\title{
In vivo optical coherence tomography of a mouse model of spontaneous ovarian cancer
}

Sawyer, Travis, Koevary, Jennifer, Rice, Photini F., Barton, Jennifer

Travis W. Sawyer, Jennifer W. Koevary, Photini F. S. Rice, Jennifer K. Barton, "In vivo optical coherence tomography of a mouse model of spontaneous ovarian cancer," Proc. SPIE 11073, Clinical and Preclinical Optical Diagnostics II, 110731E (19 July 2019); doi: 10.1117/12.2525611 


\title{
In Vivo Optical Coherence Tomography of a Mouse Model of Spontaneous Ovarian Cancer
}

\author{
Travis W. Sawyer ${ }^{1}$, Jennifer W. Koevary ${ }^{2}$, Photini F. S. Rice ${ }^{2}$, Jennifer K. Barton ${ }^{1,2}$ \\ ${ }^{1}$ College of Optical Sciences, The University of Arizona, Tucson 85721, Arizona, USA \\ ${ }^{2}$ Department of Biomedical Engineering, The University of Arizona, Tucson 85721, Arizona, USA \\ tsawyer9226@email.arizona.edu
}

\begin{abstract}
Ovarian cancer is the deadliest gynecologic cancer, but can be addressed with early detection. We investigate optical coherence tomography for imaging ovarian cancer, finding that tissue changes can be detected through quantitative analysis.
\end{abstract}

OCIS codes: $170.2520,170.6935$.

\section{Introduction}

Ovarian cancer is a devastating disease with high mortality rates due to non-specific symptoms and lack of an effective screening test, leading to frequent late diagnosis. However, if ovarian cancer is found and treated before metastasis, the 5year survival rate is $94 \%$ (versus $28 \%$ for metastatic disease). Thus, a reliable early-detection technique could greatly reduce the burden and mortality of the disease. Optical coherence tomography (OCT) is an interferometric imaging technique first introduced in 1991 [1] that yields depth-resolved, high-resolution images of tissue, providing information about the tomography and microstructure. Among other applications, OCT has shown great potential for differentiating cancerous and healthy tissue in the ovaries. [2] However, little work has investigated how features derived from OCT images change throughout disease onset. In this study, we image a mouse model that spontaneously develops ovarian cancer to evaluate OCT for detecting changes in ovarian tissue with disease onset. Imaging is done in vivo using OCT at two time points for both healthy and diseased animals, as well as those treated to undergo menopause. We find that texture features derived from OCT images show statistically significant differences between experimental groups, indicating that texture analysis and OCT have potential for assessing ovarian tissue health.

\section{Methods}

\subsection{Mouse Model}

For this experiment, we used a transgenic mouse model in which females spontaneously develop bilateral epithelial ovarian cancer. The TgMISIIR-TAg (TAg) mouse was obtained from Dr. Denise Connolly and colleagues at Fox Chase Cancer Center [3]. At 4 weeks, TAg animals may have microscopic disease and by 8 weeks all TAg animals have disease that may be either microscopic, or slight ovarian enlargement. Female offspring of both geneotypes were injected with Vehicle (sesame oil; SO) or 4-Vinylcyclohexene diepoxide (VCD) dissolved in SO at a concentration of $80 \mathrm{mg} / \mathrm{kg}$ for 20 days beginning at post-natal day seven. VCD was used to destroy preantral follicles, mimicking a post-menopausal state. Mice were imaged in vivo at both four and eight weeks of age, before being euthanized. 15 mice were imaged at both time points.

\subsection{Imaging and Analysis}

Three dimensional OCT imaging was completed with a swept source OCT system (OCS1050SS, Thorlabs). The system operates in non-contact mode with a central wavelength of $1040 \mathrm{~nm}$ and spectral bandwidth of $80 \mathrm{~nm}$. The axial scan rate was $16 \mathrm{kHz}$ and the power on the sample was measured as $0.36 \mathrm{~mW}$. The system was set to average 4 axial scans. The OCT system has $11 \mu \mathrm{m}$ transverse resolution and $9 \mu \mathrm{m}$ axial resolution in tissue. Imaging volume was ( $x$ lateral) $4 \mathrm{~mm} \times$ (y lateral) $4 \mathrm{~mm} \times(z$ axial) $2 \mathrm{~mm}$ deep and $750 \times 752 \times 512$ pixels (pixel size of approximately $5 \mu \mathrm{m} \times 5 \mu \mathrm{m}$ ). The image volume was exported as a series of $2 \mathrm{D}$ en face $(x-y)$ images or slices, and saved to disk as .tif image files.

Morphological features were extracted from OCT images using texture analysis based on the application of the greylevel co-occurrence matrix (GLCM) and also by quantifying the spatial frequency content using the Fourier transform 
(FT) [4]. The cumulative frequency distribution as a function of radial frequency was quantified by fitting to the equation $y=\alpha x^{\beta}$, where $y$ is the cumulative energy and $x$ is the radial frequency. This formula was empirically found to fit the distribution well. Thirteen texture features [5] were extracted and combined with the two frequency-based features $(\alpha, \beta)$ to yield a total of fifteen features. Statistical significance was tested using a linear mixed effects model with a random intercept accounting for the within-subject dependence and three-way interactions between age, genotype and treatment. Each group was treated independently (i.e, not pairwise) for the analysis.

\section{Results}

Analysis of OCT images in 3D shows significant differences between genotype for 8 week SO mice for seven GLCM features including Difference Entropy and Contrast (Fig. 1a). These features were found to be uncorrelated, indicating that they are not redundant. For 2D analysis, several GLCM features show significant changes between genotype (Fig. $1 b$ ); however, these have less statistical power than 3D analysis. This is consistent with our previous studies comparing 2D and 3D texture analysis [4]. Furthermore, significance was observed with the spatial frequency content between WT and TAg ovaries at both 4 and 8 weeks of age (Fig. 1c), which appears to be attributable to more high frequency content in TAg animals. VCD dosing has minimal effect of FT energy distribution at 4 weeks, but at 8 weeks there is a shift in frequency content to lower frequencies (significant for TAg animals). Both VCD dosing and age produce significant changes in multiple GLCM features, indicating that these may be a confounding factor.
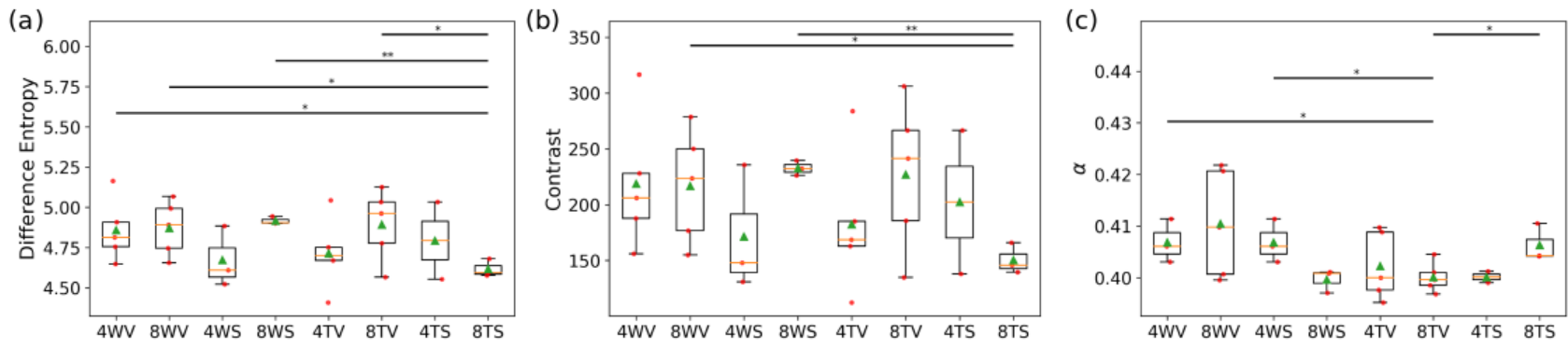

Fig. 1. Example GLCM features in 3D (a) and in 2D (b) show significant differences between TAg and Wild type animals, as do frequency-based texture features (c). Statistical significance is denoted with $*$ for $\mathrm{p}<0.05$, and $* *$ for $\mathrm{p}<0.001$. For the groups, $4 / 8$ refers to age, W/T refers to wild type or $\mathrm{TAg}$, and S/V refers to dosing with sesame oil or VCD.

\section{Conclusion}

We demonstrate with an in vivo study that OCT can be used to detect tissue changes in a mouse model of ovarian cancer. In particular, we demonstrated that the texture features derived from the grey-level co-occurrence matrix shows significant differences between control and diseased mouse groups. This indicates that OCT have high potential for assessing ovarian tissue health. Next steps include testing this modality in vivo in a clinical study, and also investigating other approaches for image analysis. However, there still remains a challenge to minimize motion artifact to maximize the data quality.

\section{References}

1. Huang D, et al. 1991 Optical coherence tomography. Science 254(5035): 1178-81.

2. Hariri L P, et al. 2010 Simultaneous optical coherence tomography and laser induced fluorescence imaging in rat model of ovarian carcinogenesis. Cancer Biol Ther 10(5): 438-47.

3. Connoly D C, et al. 2003 Female mice chimeric for expression of the simian virus $40 \mathrm{TAg}$ under control of the MISIIR promoter develop epithelial ovarian cancer. Cancer Res. 63(6):1389-97.

4. Sawyer T W, et al. 2018 Three-dimensional texture analysis of optical coherence tomography images of ovarian tissue. Phys Med Biol. 63235020.

5. Haralick R, Shanmugam K, Dinstein I. 1972 Textural features for image classication. IEEE Trans. Syst., Man, Cybern. 3(6): 610-21. 